\title{
Authorship Correction: A Community-Based Short Message Service Intervention to Improve Mothers' Feeding Practices for Obesity Prevention: Quasi-Experimental Study
}

Hong Jiang ${ }^{1,2^{*}}$, PhD; Mu Li ${ }^{3,4^{*}}, \mathrm{PhD} ; \mathrm{Li}$ Ming Wen ${ }^{3,5}, \mathrm{PhD}$; Louise Baur ${ }^{3,6}$, PhD; Gengsheng He ${ }^{1,2}$, PhD; Xiaoying $\mathrm{Ma}^{1,2}, \mathrm{MS} ; \mathrm{Xu} \mathrm{Qian}^{1,2}, \mathrm{PhD}$

\footnotetext{
${ }^{1}$ School of Public Health, Global Health Institute, Fudan University, Shanghai, China

${ }^{2}$ Key Lab of Health Technology Assessment, National Health Commission of the People's Republic of China, Fudan University, Shanghai, China

${ }^{3}$ School of Public Health, University of Sydney, Sydney, Australia

${ }^{4}$ China Studies Centre, University of Sydney, Sydney, Australia

${ }^{5}$ Health Promotion Unit, Sydney Local Health District, Sydney, Australia

${ }^{6}$ Discipline of Child \& Adolescent Health, University of Sydney, Sydney, Australia

* these authors contributed equally
}

\section{Corresponding Author:}

Hong Jiang, PhD

School of Public Health

Global Health Institute

Fudan University

175 Mailbox

138 Yixueyuan Road

Shanghai, 200032

China

Phone: 862164179976

Fax: 862164179976

Email: h_jiang@fudan.edu.cn

\section{Related Article:}

Correction of: http://mhealth.jmir.org/2019/6/e13828/

(JMIR Mhealth Uhealth 2019;7(7):e15046) doi: 10.2196/15046

The authors of "A Community-Based Short Message Service Intervention to Improve Mothers' Feeding Practices for Obesity Prevention: Quasi-Experimental Study" (JMIR Mhealth Uhealth 2019;7(6):e13828) wish to change the order of the authors on the publication so that Xu Qian is listed last.

The previous order of authorship was as follows:

Hong Jiang, Mu Li, Li Ming Wen, Louise Baur, Gengsheng He, Xu Qian, Xiaoying Ma

The correct order of authorship is as follows:

Hong Jiang, Mu Li, Li Ming Wen, Louise Baur, Gengsheng He, Xiaoying Ma, Xu Qian

Additionally, the affiliations listed for author Gengsheng $\mathrm{He}$ have also been updated. Previously, only affiliation 2 was listed for this author:

\begin{abstract}
${ }^{2}$ Key Lab of Health Technology Assessment, National Health Commission of the People's Republic of China, Fudan University, Shanghai, China
\end{abstract}

The revised listing identifies that Gengsheng He is associated with affiliations 1 and 2:

\section{${ }^{1}$ School of Public Health, Global Health Institute, Fudan University, Shanghai, China \\ ${ }^{2}$ Key Lab of Health Technology Assessment, National Health Commission of the People's Republic of China, Fudan University, Shanghai, China}

The corrections will appear in the online version of the paper on the JMIR website on July 18, 2019, together with the publication of this correction notice. Because this was made after submission to PubMed, PubMed Central, and other full-text repositories, the corrected article also has been resubmitted to those repositories. 
Edited by N Duguay; this is a non-peer-reviewed article. Submitted 15.06.19; accepted 17.06.19; published 18.07.19.

Please cite as:

Jiang H, Li M, Wen LM, Baur L, He G, Ma X, Qian X

Authorship Correction: A Community-Based Short Message Service Intervention to Improve Mothers' Feeding Practices for Obesity

Prevention: Quasi-Experimental Study

JMIR Mhealth Uhealth 2019;7(7):e15046

URL: http://mhealth.jmir.org/2019/7/e15046/

doi: $10.2196 / 15046$

PMID: 31322131

CHong Jiang, Mu Li, Li Ming Wen, Louise Baur, Gengsheng He, Xiaoying Ma, Xu Qian. Originally published in JMIR Mhealth and Uhealth (http://mhealth.jmir.org), 18.07.2019. This is an open-access article distributed under the terms of the Creative Commons Attribution License (https://creativecommons.org/licenses/by/4.0/), which permits unrestricted use, distribution, and reproduction in any medium, provided the original work, first published in JMIR mhealth and uhealth, is properly cited. The complete bibliographic information, a link to the original publication on http://mhealth.jmir.org/, as well as this copyright and license information must be included. 